\title{
Activity-Dependent Recruitment of Extrasynaptic NMDA Receptor Activation at an AMPA Receptor-Only Synapse
}

\author{
Beverley A. Clark and Stuart G. Cull-Candy \\ Department of Pharmacology, University College London, London WC1E 6BT, United Kingdom
}

\begin{abstract}
We have identified an excitatory synapse in cerebellar molecular layer interneurons at which the level of presynaptic activity determines the receptor type involved in the postsynaptic response. When small numbers of parallel fibers are activated, EPSCs are mediated solely by AMPA receptors (AMPARs), despite our finding that NMDA receptors (NMDARs) are present in the dendrites of these cells. The EPSC kinetics are fast $(\tau$ decay $=0.82 \pm 0.05 \mathrm{msec}$ at room temperature), consistent with the role these interneurons are thought to play in precisely timed inhibitory control of Purkinje cells. NMDARs are activated only when glutamate release is increased either by facilitation with brief high-frequency trains or by recruiting more presynaptic fibers with higher stimulus intensities. Under these conditions, EPSCs consist of a fast-rising AMPAR-mediated current followed by a slow component mediated by both NMDARs and AMPARs. Inhibitors of glutamate transport increase the
\end{abstract}

amplitude and prolong the time course of the compound EPSCs. In contrast, the properties of fast AMPAR EPSCs resulting from the activation of few inputs remain unchanged when glutamate uptake is blocked. Our results suggest that, at these synapses, the postsynaptic density contains AMPARs alone. It is only when transmitter release is high enough for glutamate to diffuse to the extrasynaptic space and to reach concentrations sufficient to activate extrasynaptic receptors that NMDARs are involved in the postsynaptic response. We suggest that such a spatial separation of receptor types may provide a mechanism for rapid changes in EPSC properties, depending on the amount of synaptic activity.

Key words: extrasynaptic NMDA receptor; AMPA-EPSC; diffusion; glutamate transporters; parallel fiber; cerebellar interneuron
At central glutamatergic synapses, basic properties of EPSCs are determined by the receptor subtypes present and by the amplitude and spatiotemporal profile of the transmitter concentration transient within the synaptic cleft. Functional and immunocytochemical studies have shown that many central excitatory synapses possess both AMPA receptors (AMPARs) and NMDA receptors (NMDARs) in their postsynaptic density (PSD) (Jones and Baughman, 1991; Kharazia et al., 1996; Takumi et al., 1999) and that these are coactivated by the release of a quantum of neurotransmitter (Bekkers and Stevens, 1989; Silver et al., 1992; Umemiya et al., 1999). The relative contribution of AMPARs and NMDARs to the EPSC reflects not only their different biophysical properties (including the voltage-dependent block of NMDARs by $\mathrm{Mg}^{2+}$ ) but also differences in receptor density and affinity.

It is well established that EPSCs are mediated by NMDARs alone at several types of central synapses ("silent synapses"; Malinow et al., 2000). In contrast, there are very few examples of synapses in which EPSCs are mediated solely by AMPARs. "AMPAR-only" EPSCs have been described so far in cultured retinal ganglion cells (Taschenberger et al., 1995) and at excitatory synaptic inputs to Purkinje cells in cerebellar slices (Perkel et al., 1990; Llano et al., 1991). The observation that Purkinje cells

\footnotetext{
Received Dec. 19, 2001; revised March 22, 2002; accepted March 22, 2002.

This work was supported by The Wellcome Trust. We are grateful to Keiko Shimamoto (Suntory Institute for Bioorganic Research) for the generous gift of TBOA. We thank Steven Brickley, Mark Farrant, Michael Häusser, and Arnd Roth for helpful comments on this manuscript.

Correspondence should be addressed to S. G. Cull-Candy, Department of Pharmacology, University College London, Gower Street, London WC1E 6BT, UK. E-mail: s.cull-candy@ucl.ac.uk or b.clark@ucl.ac.uk.

Copyright (ㄷ) 2002 Society for Neuroscience $0270-6474 / 02 / 224428-09 \$ 15.00 / 0$
}

from rats older than $\sim$ P14 lack functional NMDARs supports this finding (Farrant and Cull-Candy, 1991; Momiyama et al., 1996). We were interested to determine whether AMPAR-only synapses occur in neurons that do express functional NMDARs. The properties of NMDARs make it likely that, even at low density, extrasynaptic NMDARs will produce a detectable current if conditions arise whereby glutamate escapes the synaptic cleft.

Isolated somatic patches from molecular layer interneurons of the cerebellum contain both NMDAR and AMPARs (CullCandy et al., 1998; Clark and Cull-Candy, 1999; Liu and CullCandy, 2000). However, it has been reported that the NMDARmediated component of evoked EPSCs is small at parallel fiber (PF)-to-interneuron synapses (Glitsch and Marty, 1999). Whether this weak NMDAR-component arose from direct activation of synaptic receptors or from diffusion of glutamate to extrasynaptic sites is not known. However, it is clear that high-frequency, high-intensity trains of PF stimulation can activate EPSCs with large AMPAR and NMDAR components (Carter and Regehr, 2000). We have investigated whether this NMDAR component arises from synaptic or extrasynaptic receptors and how it is influenced by synaptic activity. We find that low-frequency activation of small numbers of PFs generates EPSCs mediated solely by AMPARs. Synchronous activation of larger numbers of synapses, or facilitation of glutamate release by stimulus trains, results in NMDAR activation that is enhanced by glutamate transporter blockers. We suggest that transmitter released from one or a few PF terminals fails to activate NMDARs because they are not colocalized with AMPARs in the PSD in these cells. Facilitation or overlap of glutamate released from multiple adjacent synapses activates extrasynaptic AMPARs and NMDARs. 
This results in a switch in the EPSC from AMPAR-only, to one containing both AMPAR- and NMDAR-mediated components, providing a mechanism for rapid changes in EPSC properties, depending on the intensity of synaptic activity.

\section{MATERIALS AND METHODS}

Coronal or transverse cerebellar slices $(200 \mu \mathrm{m})$ were cut from 18-d-old Sprague Dawley rats, as described previously (Barbour et al., 1994). Slicing and incubation solutions were adapted from Mann-Metzer and Yarom (1999) and consisted of (in mM): $85 \mathrm{NaCl}, 2.5 \mathrm{KCl}, 0.5 \mathrm{CaCl}_{2}, 4$ $\mathrm{MgCl}_{2}, 25 \mathrm{NaHCO}_{3}, 1.25 \mathrm{NaH}_{2} \mathrm{PO}_{4}, 75$ sucrose, and 25 glucose, bubbled with $95 \% \mathrm{O}_{2}$ and $5 \% \mathrm{CO}_{2}$. Slices were maintained at $32^{\circ} \mathrm{C}$ for $1 \mathrm{hr}$, and the slicing solution was gradually replaced $(1.3 \mathrm{ml} / \mathrm{min})$ with extracellular solution containing (in mM): $125 \mathrm{NaCl}, 2.5 \mathrm{KCl}, 2 \mathrm{CaCl}_{2}, 1 \mathrm{MgCl}_{2}, 25$ $\mathrm{NaHCO}_{3}, 1.25 \mathrm{NaH}_{2} \mathrm{PO}_{4}$, and 25 glucose. Slices were transferred to the recording chamber and, unless stated otherwise, perfused for $30 \mathrm{~min}$ (1.5 $\mathrm{ml} / \mathrm{min}$ ) with a nominally $\mathrm{Mg}^{2+}$-free extracellular solution before making recordings. All experiments were performed at $24 \pm 0.5^{\circ} \mathrm{C}$ in the presence of $100 \mu \mathrm{M}$ picrotoxin and $10 \mu \mathrm{M}$ glycine, unless stated otherwise. In experiments in which 6-cyano-7-nitroquinoxaline-2,3-dione (CNQX; Tocris Cookson, Bristol, UK) was used to block non-NMDA receptors, the glycine concentration was increased to $50 \mu \mathrm{M}$ to minimize the effect on the glycine site of the NMDA receptor (Lester et al., 1989). All chemicals were obtained from Sigma-Aldrich (Dorset, UK) unless otherwise stated.

Recording synaptic currents. Whole-cell recordings were made from visually identified stellate cells (Häusser and Clark, 1997) using an Axopatch200B amplifier (Axon Instruments, Union City, CA). Slices were viewed with an upright microscope (Axioskop FS1; Zeiss, Welwyn Garden City, UK) using infrared-differential interference contrast optics (Stuart et al., 1993). Electrodes of 3-5 M $\Omega$ were pulled from thick-walled borosilicate glass (GC-150F; Harvard Apparatus Ltd, Edenbridge, UK), coated with Sylgard resin (Dow Corning 184) and fire polished. These were filled with an intracellular solution of (in $\mathrm{mM}$ ): $125 \mathrm{CsCl}, 10$ HEPES, 10 BAPTA, 10 TEAC1, 1 QX314, $2 \mathrm{Na}_{2}$ ATP, 2 MgATP, 0.3 $\mathrm{Na}_{3}$ GTP, and $0.5 \mathrm{CaCl}_{2}$, adjusted to $\mathrm{pH} 7.25$ with $\mathrm{CsOH}$, giving a final osmolarity of $285 \pm 5 \mathrm{mOsmol} / 1$. A $10 \mathrm{~mm}$ concentration of BAPTA was included to minimize the calcium-dependent inactivation of NMDA receptors (Rosenmund et al., 1995). Series resistance was monitored continuously throughout experiments and ranged from 6 to $15 \mathrm{M} \Omega$. For series resistances $>10 \mathrm{M} \Omega$, partial compensation $(60-75 \%)$ was used by means of the amplifier compensation circuit. Data were filtered at $5 \mathrm{kHz}$ and digitized at $33 \mathrm{kHz}$. PF inputs to stellate cells were stimulated $(<200$ $\mu \mathrm{sec}, \pm$ biphasic pulses) using a patch electrode (3-5 M $\Omega$ ) placed in the molecular layer at least $100 \mu \mathrm{m}$ away from the recording electrode. Interstimulus intervals were $5-10 \mathrm{sec}$. "Low-intensity stimulation" (8-15 V) was used in some experiments. This was the threshold voltage required to activate the smallest possible number of PFs, with a failure rate of $>30 \%$. Even at this intensity, there was a significant amount of release asynchrony. To evaluate the involvement of NMDA receptors in EPSCs evoked by low-intensity stimulation, average EPSCs were constructed from events selected to avoid a contribution of release asynchrony to the EPSC time course. EPSC decays were fitted ( $t=0$ at the peak) with one or two exponentials, and the weighted tau $\left(\tau_{\mathrm{w}}\right)$ was calculated according to: $\tau_{\mathrm{w}}=\left(\tau_{1} * a_{1}\right)+\left(\tau_{2} * a_{2}\right)$, where $a_{1}$ and $a_{2}$ are the relative amplitudes of the two exponential components.

Ionophoresis of glutamate. Pipettes for ionophoresis were pulled from thin-walled borosilicate filamented glass using a Livingstone-type puller (Narishige, Tokyo, Japan). Electrodes had resistances of 30-50 M $\Omega$ when filled with $100 \mathrm{~mm}$ sodium glutamate. Current pulses of between -30 and $-90 \mathrm{nA}$ and of $5-25 \mathrm{msec}$ duration were required to eject glutamate. No retaining current was needed. To identify the dendrites and axons of interneurons and to map responses to glutamate, cells were filled via the patch electrode with the fluorescent dye Alexa Fluor 594 hydrazide $(25 \mu \mathrm{M}$; Molecular Probes, Eugene, OR). The Alexa dye was also included in the ionophoresis electrode, which by virtue of its negative charge, was ejected with the glutamate, allowing visualization of the ionophoresis electrode and the area of glutamate ejection. To quantify responses from different zones of glutamate ionophoresis, averages of at least 30 sweeps were made, aligning on the first rising point of the ionophoretic pulse.

Local perfusion of glutamate transporter blockers. Extracellular solution containing the glutamate transporter blockers DL-threo- $\beta$-benzyloxyaspartate (DL-TBOA, a generous gift from Keiko Shimamoto; Shimamoto et al., 1998) or L-trans-pyrrolidine-2,4-dicarboxylic acid (L-trans-PDC, Tocris Cookson) were applied locally using a pressure application system. Pipettes (2-3 M $\Omega$ ) pulled from thin-walled borosilicate filamented glass were used, and the pressure was adjusted to avoid movement artifacts. Using this technique, it was possible to interleave groups of sweeps of evoked EPSCs with those evoked in the presence of blockers, controlling for instability of EPSC amplitude and avoiding the effects of prolonged application of transporter blockers.

\section{RESULTS \\ Unitary EPSCs in molecular layer interneurons arise solely from AMPAR activation}

To investigate the relative contribution of both AMPA and NMDARs to synaptic transmission in molecular layer interneurons (stellate cells), we have examined spontaneous and evoked EPSCs in nominally $\mathrm{Mg}^{2+}$-free external solution. EPSCs were evoked by low-intensity stimulation (see Materials and Methods) of PFs. To be certain that any NMDAR-mediated component would be detected, EPSCs were recorded at both positive and negative holding potentials. Figure $1 A$ shows single sweeps in which EPSCs were evoked at -60 and $+50 \mathrm{mV}$ with low-intensity stimulation. No detectable NMDAR-mediated component was apparent under control conditions at either holding potential.

To minimize the contribution of asynchronous release to the mean EPSC time course, events with notched rises or multiple peaks were discarded. The averaged currents shown in Figure $1 B$ were generated by aligning EPSCs on their initial rise $(10 \%$ of full amplitude; mean 10-90\% rise time $=0.19 \pm 0.01 \mathrm{msec} ; n=$ 9). The decay of the averaged EPSCs was rapid and could be fitted with two exponential components, giving a weighted mean time constant $\left(\tau_{\mathrm{w}}\right)$ of $0.82 \pm 0.05 \mathrm{msec}(n=9)$ at $-60 \mathrm{mV}$ and $1.24 \pm 0.2 \operatorname{msec}(n=4 ; p=0.08$ paired $t$ test $)$ at $+50 \mathrm{mV}$. As is apparent from Figure $1 B$, the addition of D-AP-5 did not significantly alter the average EPSC decay at either positive or negative potentials $(0.83 \pm 0.05 \mathrm{msec}$ at $-60 \mathrm{mV} ; 1.27 \pm 0.04 \mathrm{msec}$ at +50 $\mathrm{mV} ; p=0.9)$. Furthermore, the decay time course of spontaneous $\operatorname{EPSCs}\left(\tau_{\mathrm{w}}=1.16 \pm 0.12 \mathrm{msec}\right.$ at $-60 \mathrm{mV}, 1.4 \pm 0.12 \mathrm{msec}$ at $+50 \mathrm{mV} ; n=5$ ) was not significantly different from EPSCs evoked by low-intensity stimulation. These were also unaffected by D-AP-5, yielding mean weighted decay time constants of $1.16 \pm 0.13 \mathrm{msec}$ at $-60 \mathrm{mV}$, and $1.48 \pm 0.16 \mathrm{msec}$ at $+50 \mathrm{mV}$ ( $n=6$ cells) (Fig. 1C). Because we found that the EPSCs were abolished by the non-NMDAR antagonist CNQX $(10 \mu \mathrm{M})$ and by the selective AMPAR antagonist GYKI 53655 (10-25 $\mu \mathrm{M})$ (Liu and Cull-Candy, 2000), our data indicate that at these synapses the unitary EPSCs were mediated purely by AMPAR activation.

The extrasynaptic spread of glutamate depends critically on temperature (Asztely et al., 1997). Along with a likely increased release probability (Van der Kloot and Molgo, 1994; Hardingham and Larkman 1998), the diffusion coefficient increases slightly with temperature (Hille, 1992). Counterbalancing this, uptake of glutamate by transporters increases strongly with temperature (Wadiche and Kavanaugh, 1998). We examined EPSCs at more physiological temperatures to see if any of these factors could change the contribution of NMDARs. When EPSCs were evoked by low-intensity stimulation at $35^{\circ} \mathrm{C}$, the decay times were faster than at room temperature, giving mean weighted decay time constants of $0.61 \pm 0.05 \mathrm{msec}(n=5$ cells $)$ at $-70 \mathrm{mV}$ and $0.86 \pm$ $0.15 \mathrm{msec}$ ( $n=3$ cells) at $+50 \mathrm{mV}$. Furthermore, the EPSCs remained insensitive to D-AP-5 at positive and negative potentials, confirming the absence of an NMDAR-mediated component to the EPSCs under these conditions.

Recent work has indicated that differences may occur in the 
A
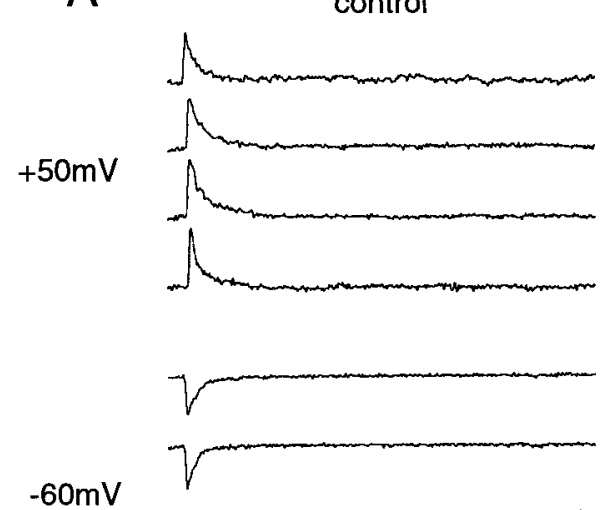

$-60 \mathrm{mV}$

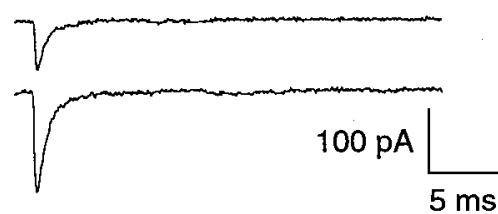

AP5
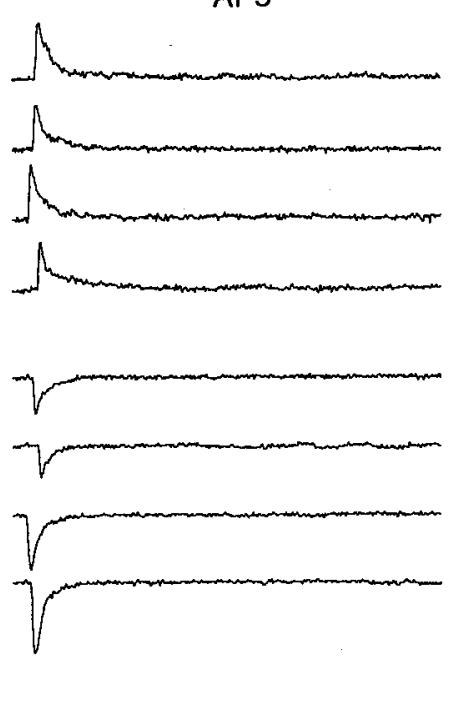

C
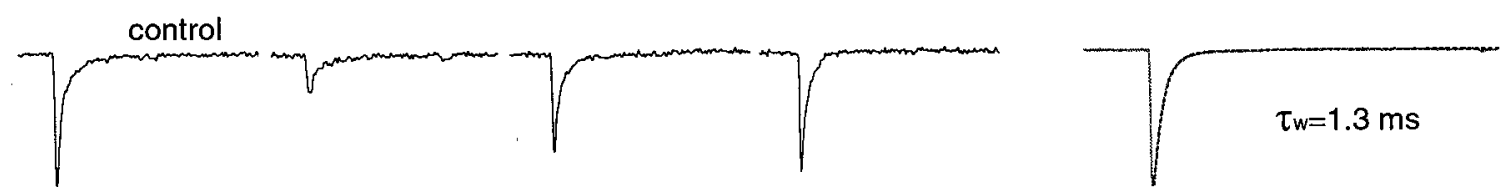

AP5
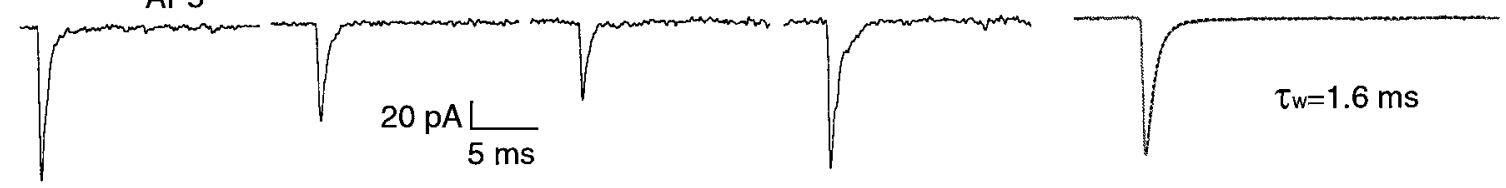

B
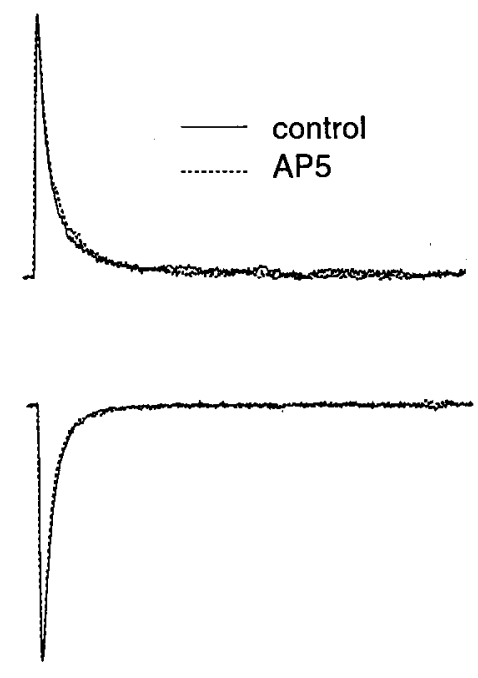

Figure 1. Spontaneous EPSCs and those evoked in interneurons by low-intensity stimulation are mediated by AMPA receptors alone. $A$, Individual sweeps in which EPSCS were successfully evoked by threshold stimulation of the parallel fibers. EPSCs under control conditions (left) and in the presence of $50 \mu \mathrm{M}$ D-AP-5 (right) are shown at holding potentials of -60 and $+50 \mathrm{mV}$. The stimulus artifact is lost because of the restricted time window shown. $B$, Overlay of averages of $>30$ sweeps in both conditions shows that NMDA receptor antagonists have no effect on the EPSC time course. $C$, Examples of consecutive single spontaneous EPSCs (sEPSCs) occurring in an interneuron recorded at $-60 \mathrm{mV}^{\text {in nominally Mg}}{ }^{2+}$-free conditions. The normalized average EPSCs are shown on the far right with the fitted biexponential function indicated by the dotted line. Addition of $50 \mu \mathrm{M} \mathrm{D-AP-5}$ (bottom row) had no effect on the sEPSC, and there was no significant difference in the fitted average.

synaptic and extrasynaptic distribution of receptors containing the NR2A subunit (Rumbaugh and Vicini, 1999; Tovar and Westbrook, 1999). NR2A-containing receptors can be observed exclusively at synaptic locations during synaptic maturation, whereas extrasynaptic receptors do not contain this subunit. The glycine sensitivity of NMDARs is dependent on receptor subunit composition, with NR1/NR2A receptors requiring a high concentration of glycine to be fully activated (Kutsuwada et al., 1992). One possible explanation for the lack of an NMDARmediated component in unitary EPSCs in interneurons might be that the glycine concentration used $(10 \mu \mathrm{M})$ was insufficient for activation of any NR2A-containing receptors that might be present at PF-interneuron synapses. We compared thresholdevoked EPSCs in the presence of 10 and $50 \mu \mathrm{M}$ glycine in nominally $\mathrm{Mg}^{2+}$ free solution. In five cells tested, no difference was found between EPSCs evoked under the two conditions. An example of this is illustrated in Figure 2.

\section{NMDARs are present on the dendrites of molecular layer interneurons}

There is evidence that spillover of glutamate following bulk activation of PFs can activate NMDARs in molecular layer interneurons (Carter and Regehr, 2000). However, it is not known whether these receptors are located on the dendrites or mainly on the soma and axon. The possibility that at least some NMDARs are present in the axon is supported by the demonstration that direct application of NMDA onto these neurons modulates the release of GABA from their axon terminals (Glitsch and Marty, 1999). To assess whether NMDARs are present on the dendrites of molecular layer interneurons and hence near to synaptic inputs or whether the lack of involvement of NMDARs in the generation of unitary EPSCs reflected their absence from the dendrites, we tested the response of these cells to glutamate applied selectively to different segments of the same neuron (in the presence of $10 \mu \mathrm{M}$ CNQX). While recording from the soma of interneurons filled with the fluorescent dye Alexa Fluor 594, we mapped NMDAR responses by applying $25 \mathrm{msec}$ ionophoretic pulses of glutamate onto the dendrites, soma, and axon.

The results of such an experiment are depicted in Figure 3. The top panel (Fig. $3 A$ ) shows the soma and dendrites of a filled interneuron; the lower panel shows part of the axon at higher magnification. Figure $3 B$ shows the responses obtained when glutamate was applied at the dendritic, somatic, and axonal locations indicated by the arrows in Figure $3 A$. The largest responses were invariably obtained from the soma, but responses were also 
A
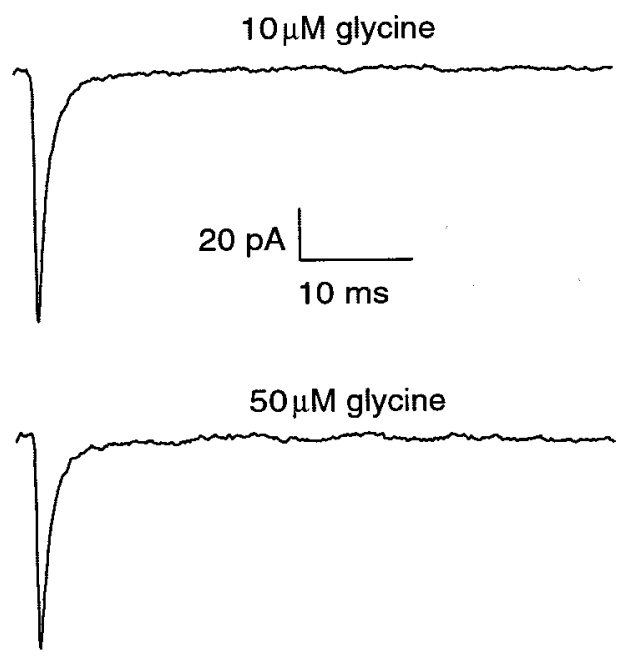

B

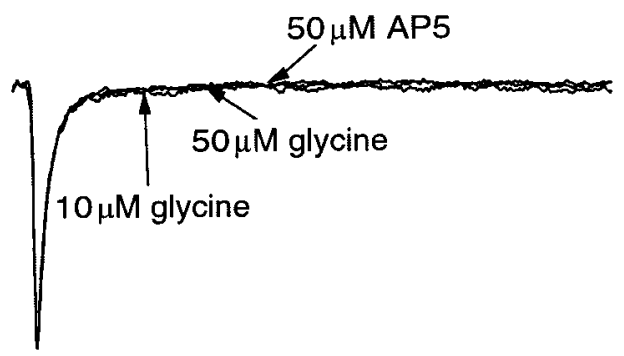

Figure 2. An NMDAR-mediated component is not revealed by increased glycine concentration $A$, Average responses in an interneuron to low-intensity parallel fiber stimulation at $0.5 \mathrm{~Hz}$ in the presence of $10 \mu \mathrm{M}$ (top panel) and $50 \mu \mathrm{M}$ (bottom panel) glycine at a holding potential of -60 $\mathrm{mV}$. $B$, Overlay of normalized average EPSCs under the two different glycine concentrations and after addition of D-AP-5 shows that no NMDAR current was revealed when glycine concentration was increased.

readily obtained from the dendrites. No current was recorded when glutamate was ejected onto the axon $(n=4$ cells, each axon was tested at multiple locations) or when the ionophoretic pipette was moved $\sim 20 \mu \mathrm{m}$ above the focal plane of the cell. These experiments indicate that, although not involved in generation of unitary EPSCs, NMDARs are present on the soma and dendrites of molecular layer interneurons. This suggests that they are distant from the sites of glutamate release and therefore are not colocalized with the AMPARs that mediate the unitary EPSC. The lack of response from the axon suggests that any NMDARs present on the axon terminals occur at low density and that the current evoked is too small to be resolved during somatic recording.

\section{The contribution of NMDARs to EPSCs depends on the number of parallel fibers activated}

The AMPAR-mediated EPSCs described above were evoked after the activation of a small number of PFs. Although the duration of the synaptic glutamate transient and the distance over which glutamate travels from its release site are not known, both of these parameters are likely to be increased if release occurs simultaneously at several closely spaced synapses (Otis et al., 1996; Barbour and Häusser, 1997; Rusakov et al., 1999). We therefore next investigated whether activating a greater number of $\mathrm{PF}$ axons could influence the type of receptors that contribute to the EPSC.

Figure $4 A$ shows EPSCs produced by activating a progressively

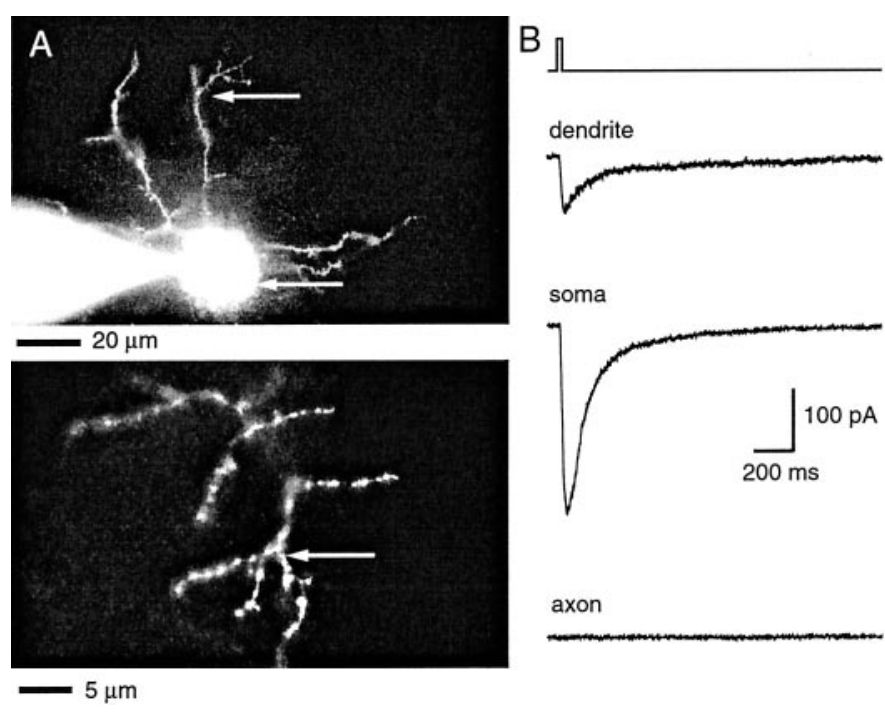

Figure 3. Ionophoretic mapping of NMDAR-mediated responses in a molecular layer interneuron. $A$, Sagittal view of an interneuron filled with Alexa Fluor 594 during whole-cell patch recording. The dendrites and soma are shown in the top panel, and a section of the axonal arbor is shown in the bottom panel. The locations of glutamate application by ionophoresis are indicated by the arrows. B, Averages of 25 currents recorded in response to $25 \mathrm{msec}$ pulses of glutamate $(100 \mathrm{~mm}$ in electrode) ejected onto the dendrites, soma, and axon of the illustrated cell. Holding potential was $-60 \mathrm{mV}$, and the antagonists TTX $(0.5 \mu \mathrm{M})$, picrotoxin $(100 \mu \mathrm{M})$, and CNQX $(10 \mu \mathrm{M})$ were present in nominally $\mathrm{Mg}^{2+}$-free solution.

larger number of PFs. This was achieved by increasing the stimulus strength from its initial "low intensity" value by $10 \mathrm{~V}$ increments. In Figure $4 A$, the initial low-intensity stimulus $(10 \mathrm{~V})$ and the first incremental increase $(20 \mathrm{~V})$, evoked an EPSC that was abolished by CNQX. However, it is apparent that at higher stimulus intensities a small, slowly decaying component remained. This residual current was blocked by D-AP-5 (50 $\mu \mathrm{M}$; data not shown) indicating that it was mediated by NMDARs. Figure $4 B$ shows the relationship between stimulus intensity and the total charge carried by AMPAR and NMDAR channels (five cells). The NMDAR-mediated component was apparent only at stimulus strengths greater than approximately twice the threshold intensity. There was no significant NMDAR-mediated response at the first incremental increase in stimulus strength (charge not significantly different from zero; $p=0.4$ ), showing that synchronous release of glutamate from a larger number of PFs, than was activated using threshold stimulation, was required to activate NMDARs in these cells. Given that NMDARs exhibit a higher apparent affinity for glutamate than do AMPARs, this observation suggests that the NMDARs are distant from release sites and are activated only by pooling of transmitter from multiple active synapses. This idea is supported by the fact that the rise time for the NMDA component was relatively slow (time-to-peak, $40 \pm$ 4.9 msec; $n=9$ cells), which may also reflect the lower concentration of glutamate present at the extrasynaptic receptors (Clements and Westbrook, 1991; Haas et al., 1998).

\section{Facilitation of glutamate release from a small number of parallel fibers can activate NMDARs}

Although dendritic NMDARs in these interneurons can be activated by glutamate released by large numbers of PFs, in principle, if release was facilitated, these receptors should also be activated during stimulation of a small number of fibers. To address this 

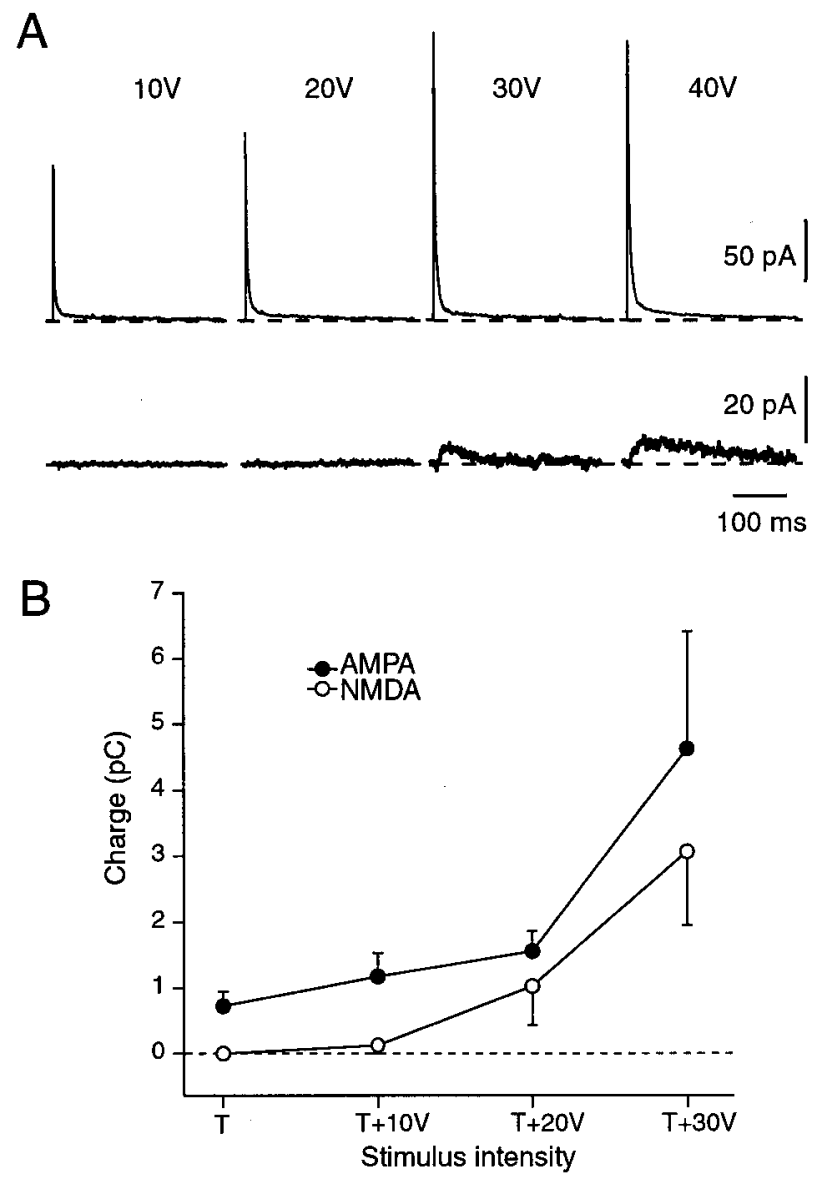

Figure 4. Increased synaptic activity results in a compound EPSC mediated by both AMPA and NMDA receptors. $A$, Mean EPSCs (averages of 25-50 sweeps) resulting from parallel fiber stimulation using the different stimulus intensities shown $\left(V_{\text {hold }}=+50 \mathrm{mV}\right)$. Top traces are controls, and bottom traces are in the presence of $10 \mu \mathrm{M} \mathrm{CNQX}$ (note different calibration), showing the appearance of an NMDA component at $30 \mathrm{~V}$ stimulus intensity. $B$, Summary of charge carried by AMPAR and NMDAR channels with respect to stimulus intensity $(n=4)$. Values for AMPAR-mediated charge transfer were obtained by subtracting values in CNQX from those in control conditions. T, Threshold low-intensity stimulation.

issue we examined the effect of trains of stimuli, which have been shown to enhance glutamate release without recruiting additional PFs (Kreitzer and Regehr, 2000). Specifically, we investigated the frequency dependence of NMDAR activation by using suprathreshold stimulation intensities, which were just sufficient to avoid failures of the fast AMPAR-mediated EPSCs.

After the addition of $10 \mu \mathrm{M}$ CNQX, we applied bursts of four stimuli $(0.5-100 \mathrm{~Hz})$ to PFs. As shown in Figure $5 A$, an NMDAR-mediated component was activated at stimulus frequencies of $\geq 6.25 \mathrm{~Hz}$. The size of this current varied considerably from cell to cell, particularly at longer interstimulus intervals when its amplitude was difficult to quantify. We therefore integrated the average current to obtain the charge transfer. Figure $5 A$ shows typical examples of these current integrals (smooth line) at the frequencies tested, overlaid with the averaged currents in which four stimulus artifacts indicate the timing of the stimuli. The mean charge transfer from seven cells is summarized in Figure $5 B$. Values have been normalized to those obtained at 100 Hz. The NMDAR charge transfer increased steeply in the lower frequency range and had still not reached a plateau at $100 \mathrm{~Hz}$

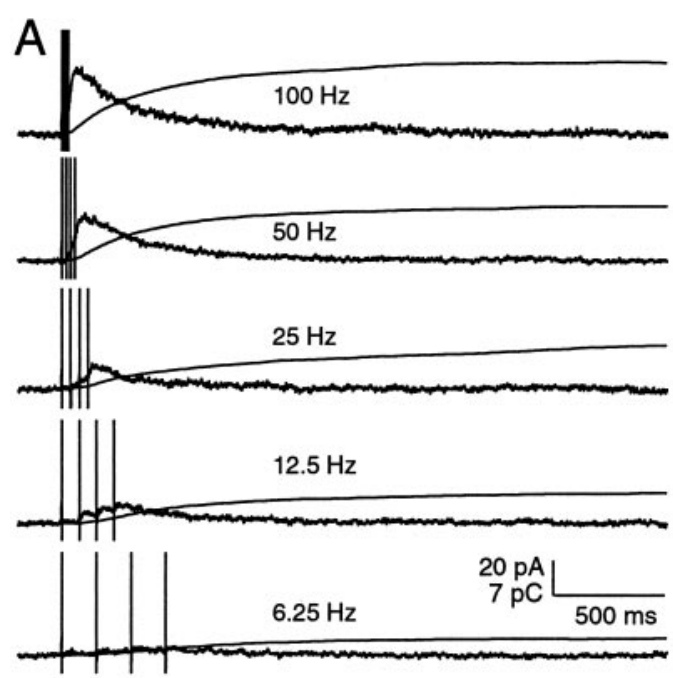

B

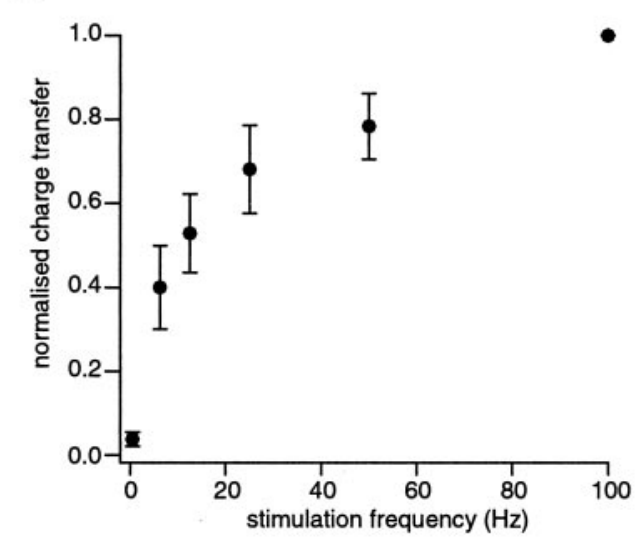

Figure 5. Frequency dependence of NMDA receptor-mediated EPSCs. $A$, Trains of four parallel fiber stimuli evoked CNQX-insensitive currents in interneurons voltage clamped at $+50 \mathrm{mV}$, which increased in amplitude with increasing frequency of stimulation. The current integral (smooth line) is superimposed on the mean synaptic currents (averages of 25-30 sweeps). $B$, Charge transfer normalized to that obtained at $100 \mathrm{~Hz}$ is represented with respect to stimulation frequency for seven cells. Error bars indicate SEM.

stimulation. These experiments indicate that a modest increase in action potential firing frequency, arising within a small number of fibers, is sufficient to change the synaptic response from AMPARonly to one mediated both by AMPARs and NMDARs at these synapses.

\section{The influence of glutamate transporters on NMDAR contribution to EPSCs}

Inhibitors of glutamate transporters have been shown previously to have little effect on the time course of the non-NMDARmediated EPSC in molecular layer interneurons (Barbour et al., 1994). However, if the NMDAR-mediated EPSC that we observed was generated by receptors located distant from release sites, it would be expected to be enhanced in the presence of glutamate uptake blockers. We therefore examined this possibility. In the absence of synaptic stimulation, application of the transporter blocker L-trans-pyrrolidine-2,4-dicarboxylic acid (Ltrans-PDC; $100 \mu \mathrm{M})$ reduced the amplitude of spontaneous synaptic currents and generated a large inward current accompanied by a noise increase. This suggested an accumulation of glutamate 


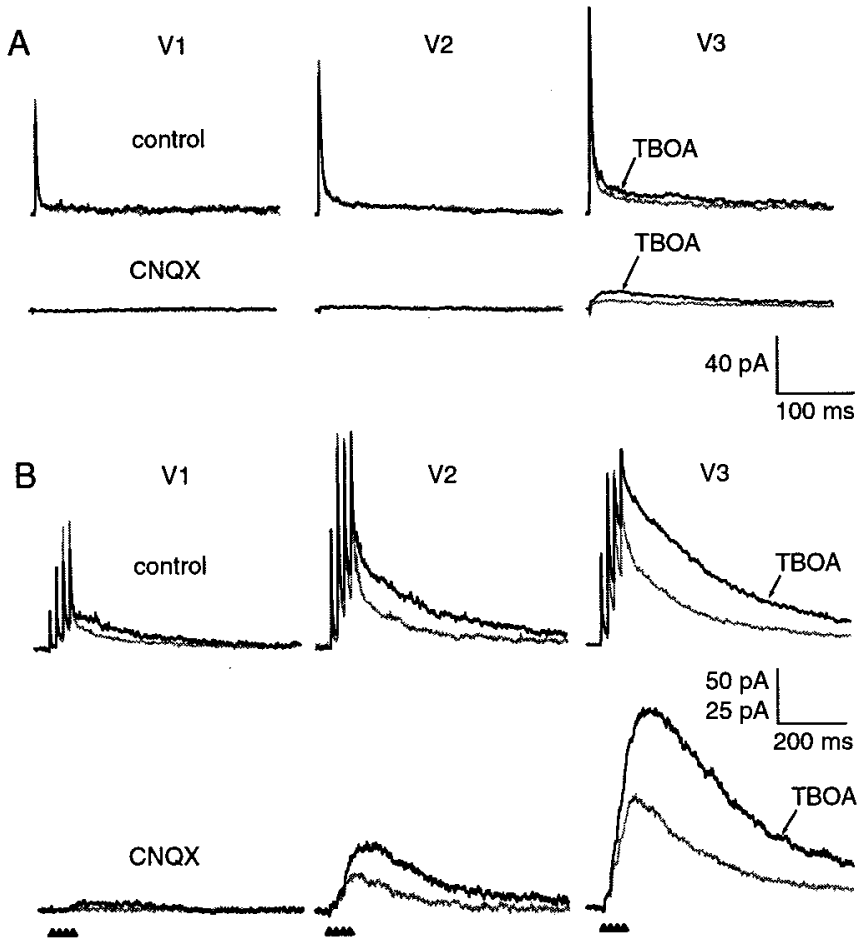

Figure 6. Effect of glutamate transporter blockade on interneuron EPSCs. $A$, Average EPSCs evoked at $0.5 \mathrm{~Hz}$ at three increasing stimulus intensities. Superimposed thin and thick traces are averaged data in the absence and presence of $50 \mu \mathrm{M}$ DL-TBOA, respectively. Top traces show the effect of DL-TBOA under control conditions, and bottom traces show the effect on the NMDAR-mediated component isolated in $20 \mu \mathrm{M}$ CNQX. $B$, Responses of the same cell as in $A$ to $50 \mathrm{~Hz}$ trains of parallel fiber stimuli. DL-TBOA application prolonged the compound EPSC train at all stimulus intensities and, in this cell, enabled activation of an NMDA component that was previously absent at V1. Stimulus artifacts have been blanked, and stimulus timing is marked by the filled triangles.

in the extracellular space, likely not only because of block of uptake but also because of glutamate release by heteroexchange triggered by the transport of L-trans-PDC (Griffiths et al., 1994; Volterra et al., 1996; Koch et al., 1999). It was therefore not possible to interpret the data obtained in the presence of this transporter blocker.

We next turned to the selective glutamate transporter blocker DL-TBOA $(50 \mu \mathrm{M})$, which is neither transported nor causes heteroexchange of glutamate (Shimamoto et al., 1998; Waagepetersen et al., 2001). In addition, we made these recordings at positive holding potentials in the presence of $1 \mathrm{mM} \mathrm{Mg}^{2+}$. This was to minimize the actions of untransported glutamate on NMDARs in other neurons in the slice. Figure 6 illustrates average data from a cell in which EPSCs were evoked at $0.5 \mathrm{~Hz}$ (Fig. 6A) and $50 \mathrm{~Hz}$ (Fig. 6B) at increasing stimulus intensities (V1-V3). DL-TBOA was applied locally from a perfusion pipette. The top traces in Figure $6, A$ and $B$, illustrate the effect of DL-TBOA on EPSCs in the absence of glutamate receptor antagonists; the bottom traces show its effect on the NMDAR component (in the presence of $5 \mu \mathrm{M}$ CNQX). From the data in the left-hand panels of Figure $6 A$, it is apparent that DL-TBOA had little effect on EPSCs evoked by low-intensity, low-frequency stimuli. However, when a greater number of PFs were activated at low frequency (V2 and V3), DL-TBOA prolonged the EPSCs. When bursts of stimuli were used to facilitate release (Fig. 6B), DL-TBOA had a small effect when the stimulus intensity was low
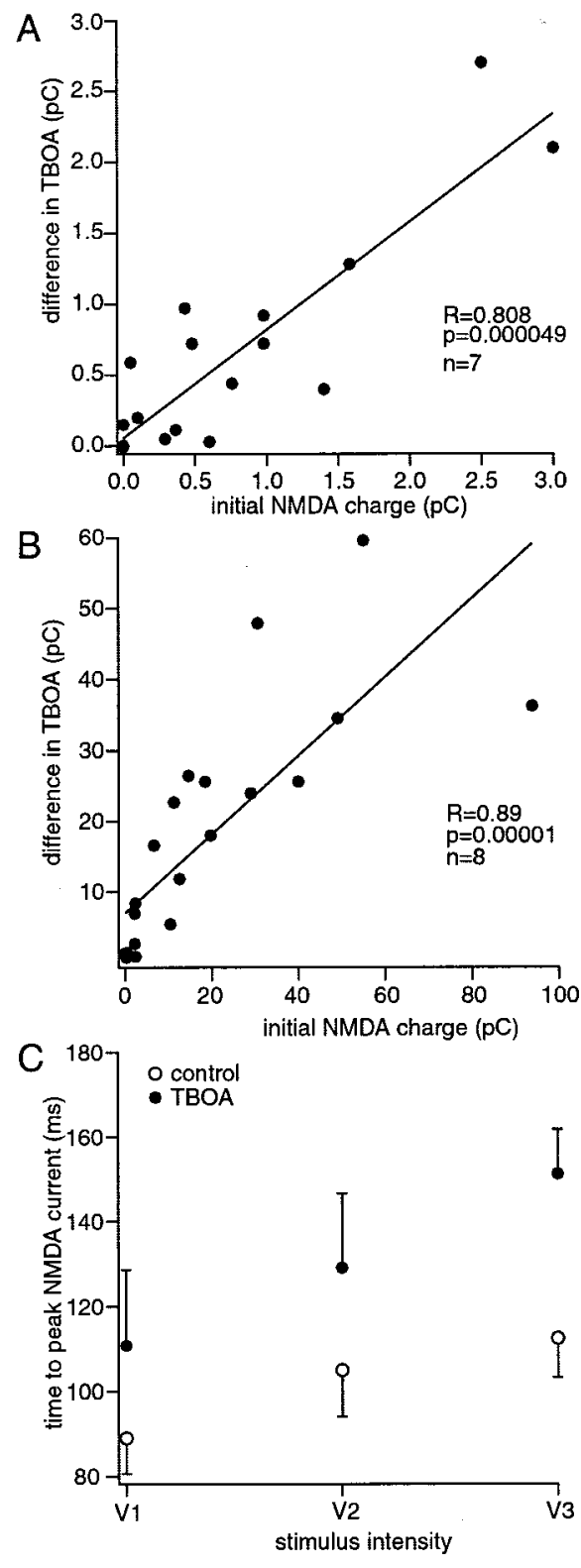

Figure 7. The effect of inhibition of glutamate transporters is correlated with the amount of glutamate released. $A$, The relationship between initial NMDAR-mediated charge resulting from a single stimulus at 0.5 $\mathrm{Hz}$ for different stimulation intensities. Each data point represents one stimulation intensity for each of the seven cells included. Correlation coefficients $(R)$ and degree of significance $(p)$ were obtained using nonparametric tests (KS). $B$, Same as $A$, but for $50 \mathrm{~Hz}$ trains. $C$, The effect of stimulus intensity on the time taken for NMDAR-mediated current resulting from $50 \mathrm{~Hz}$ trains to reach peak amplitude.

(V1), but this effect was greatly enhanced with increasing stimulus strength. The dramatic effect of DL-TBOA on the NMDARmediated component is more clearly seen in the presence of CNQX (Fig. 6B, bottom traces).

The remaining experiments on the effect of DL-TBOA on the NMDAR-mediated component of the EPSC were performed in the presence of CNQX. The results are summarized in Figure 7. The magnitude of the effect of DL-TBOA was clearly dependent on the initial NMDAR-mediated charge transfer with both 0.5 and $50 \mathrm{~Hz}$ stimulation (Fig. $7 A, B$ ). This is consistent with the idea that the effect of DL-TBOA depended on the quantity of 
glutamate released. Hence, the larger the initial charge, the greater its increase in the presence of DL-TBOA. As shown in Figure $7 C$, the time taken for the NMDA current to reach its peak (measured from the beginning of the $50 \mathrm{~Hz}$ train) depended on stimulus intensity and was prolonged by blockade of glutamate transport.

\section{DISCUSSION}

We have identified a synapse in molecular layer interneurons, at which unitary EPSCs are mediated by AMPA receptors alone, despite the observation that these cells express functional NMDARs. These NMDARs are activated by synaptically released glutamate only under conditions of intense activity. Our findings reveal a switch in functional behavior of the synapse that is regulated by the level of presynaptic activity: unitary EPSCs are mediated purely by AMPARs, whereas compound EPSCs are mediated by both AMPAR and NMDARs. Transporters regulate the extent of NMDAR activation, but only under conditions of dense PF activity. These findings identify, for the first time, a synapse in which the type of ionotropic glutamate receptor activated depends on the amount of synaptic activity.

\section{Activity dependence of glutamate receptor contribution to EPSCs}

We find that activation of a small number of PF synapses on molecular layer interneurons (such as during spontaneous synaptic release of glutamate) generates EPSCs mediated purely by AMPARs. The decay time course of these EPSCs is fast and is consistent with the deactivation time constant of AMPARs measured in outside out patches from these neurons (Barbour et al., 1994). Stimulation of larger numbers of fibers increases the EPSC amplitude and recruits an NMDAR-mediated current to the synaptic response. Because our experiments show that NMDARs are present in the dendrites of these neurons, it is likely that these receptors are involved in generating this current.

What underlies this activity-dependent difference in NMDAR involvement in synaptic transmission? Because NMDARs have a high apparent affinity for glutamate when compared with AMPARs (Lester et al., 1990; Clements and Westbrook, 1991), their activation would be expected to accompany the AMPAR-EPSC if both receptor types were present in the PSD (Clements et al., 1992; Holmes, 1995; Diamond, 2001). The most likely explanation for the lack of NMDAR contribution to the unitary response, is that, although AMPARs are present in the PSD (as indicated by the rapid AMPAR EPSC rise time; $0.19 \pm 0.01 \mathrm{msec} n=9$ 10-90\%), NMDA receptors are located outside the postsynaptic specialization and activated only after increased transmitter release.

Immunocytochemical techniques using nondiffusible markers (such as gold particles) have been used to examine synaptic localization of neurotransmitter receptors in electron microscopic sections of tissue from different brain regions. To date, no detailed comparison of AMPA and NMDAR localization has been made in the molecular layer of cerebellum. In particular, immunoreactivity for NMDARs at PF-interneuron synapses has not been described. Although immunocytochemical (Takumi et al., 1999; Racca et al., 2000) and functional approaches (Wu et al., 1996; Isaac et al., 1997; Li and Zhuo, 1998; Rumpel et al., 1998) have identified synapses in various brain regions that contain only NMDARs, most central synaptic currents are mediated both by AMPARs and NMDARs, when both are present in the cell. Indeed, available evidence suggests that the majority of AMPAR and NMDARs are concentrated in the PSD with a steep decline in concentration at the edges of the postsynaptic specialization. In contrast, our data suggest that at PF-interneuron synapses, NMDARs are not targeted to the PSD and are expressed only in the extrasynaptic membrane.

The dendrites of molecular layer interneurons are mostly aspiny, and the majority of excitatory synapses are made onto smooth dendritic membrane (Palay and Chan-Palay, 1974). In addition, glial processes are rarely observed in close proximity to these synapses (Chaudhry et al., 1995), and so diff usion out of the synaptic cleft will be less restricted in comparison with synapses made onto spines. When glutamate is released from few (one or two) synapses, the glutamate transient is likely to be short-lived, because glutamate diff uses quickly away from the release site and is diluted in the extracellular space. Under these conditions, the transmitter concentration outside the synaptic cleft is unlikely to be sufficient to activate extrasynaptic receptors. The fast decay kinetics of the pure AMPAR EPSC that we observe when few fibers are activated suggests that this is the case at PF synapses on interneurons. With dense activity of parallel fibers, glutamate may accumulate to activate extrasynaptic NMDARs, because the free diffusion between synapses would be expected to allow spillover of transmitter from adjacent boutons. In this context we use "spillover" to refer to glutamate diffusion to extrasynaptic receptors and not necessarily to receptors at neighboring synapses. Our experiments demonstrate with, stimulus trains, that frequency-dependent facilitation of glutamate release enhances this effect.

Another possible explanation of our findings may be that some PF synapses onto interneurons do contain NMDARs but that these synapses have a very low release probability $\left(P_{\mathrm{r}}\right)$ and generate EPSCs only under conditions when $P_{\mathrm{r}}$ is enhanced, such as during high-frequency activation. This possibility is difficult to address directly, because manipulations to increase $P_{\mathrm{r}}$ also greatly reduce the likelihood of evoking EPSCs from just a small number of synapses. However, we found that an increase in temperature, another condition that is expected to increase $P_{\mathrm{r}}$ (Van der Kloot and Molgo, 1994; Hardingham and Larkman 1998), did not reveal a population of synapses at which an NMDAR component contributed to EPSCs evoked by low-intensity stimulation. It is also not possible to entirely rule out that some PF synapses on interneurons may contain NMDARs, although we did not detect them in this study. If this is the case, they may contribute to the NMDAR-mediated component observed during high-intensity or high-frequency stimulus protocols. However, it is unlikely that they would be solely responsible for the NMDAR component, because such synapses are likely to be few in number, in comparison with the AMPAR-only synapses.

\section{The role of glutamate transporters in NMDA receptor activation}

We find that, during low-intensity stimulation at low frequency, block of glutamate transport does not induce an NMDAR involvement in the EPSC. However, it does increase the NMDARmediated charge transfer when glutamate release is enhanced, either by activation of more synapses or by facilitation by highfrequency bursts. This is likely to be a consequence of two factors. First, as discussed above, the spatial profile of glutamate will be different under the two conditions, with greater activity producing a much wider spread of glutamate. Second, if glutamate transporters are located distant from the synapse, uptake will be 
effective only when the glutamate transient spreads far enough to reach them.

The lack of glial processes around excitatory synapses onto cerebellar interneurons (Chaudhry et al., 1995) not only implies that the diffusional path between synapses is clear, but also that the distance from the site of glutamate release to the nearest glial glutamate transporters (GLT1 and GLAST) is long. Immunogold labeling of GLT1 and GLAST has shown that these transporters are located more distant from PF-interneuron synapses compared with PF-Purkinje cell synapses and, in addition, that they are present at lower densities (Chaudhry et al., 1995). Although there is some evidence that the neuronal transporter EAAC1 may be present in molecular layer interneurons (Furuta et al., 1997), it is not known whether this transporter is localized perisynaptically in these cells. Because transporter blockers did not affect EPSCs arising from a small number of active parallel fibers, it is probable that any neuronal glutamate transporters present are not optimally located to regulate NMDAR involvement under conditions when release is low. This result also rules out the possibility that NMDARs are perisynaptic and that synaptic glutamate transporters prevent their activation during single fiber EPSCs.

\section{Functional implications for excitation of molecular layer interneurons}

Parallel fibers (granule cell axons), are densely packed in the molecular layer of the cerebellum. Little is known about patterns of activity in vivo from the granule cells onto their targets (interneurons and Purkinje cells). Specifically, it is not known whether coincident excitatory inputs converge onto a localized area of dendrite or if they are distributed across different, distinct dendritic areas. According to our findings, the number and spatial distribution of active fibers will determine whether or not NMDARs are activated in vivo. Although dense parallel fiber activity (when many adjacent fibers are activated), will result in NMDAR activation in interneurons, it is probable that an equivalent number of sparsely distributed but coincident inputs might generate purely AMPAR-mediated EPSCs. In effect this means that the same number of active inputs can drive two different kinds of postsynaptic response, depending on the way in which they are organized.

Molecular layer interneurons spontaneously fire action potentials in vivo (Armstrong and Rawson, 1979) and in slice preparations. This provides a continuous but irregular barrage of inhibitory input to both Purkinje cells and other interneurons, which modulates integration of postsynaptic potentials (Häusser and Clark, 1997). In addition, synaptically driven action potentials in interneurons are thought to provide specifically targeted inhibitory inputs that shape the response of Purkinje cells to excitation (Eccles et al., 1967). The presence of AMPAR-only synapses that generate EPSCs of relatively fast kinetics will allow temporally precise coupling of single EPSCs to action potentials and thus enable accurately timed inhibitory output. This behavior would be consistent with emerging principles of interneuron function (Geiger et al., 1997).

We have found previously that AMPARs at PF-interneuron synapses can be $\mathrm{Ca}^{2+}$-permeable when parallel fibers are stimulated at low frequency (Liu and Cull-Candy, 2000). Little is known about the characteristics of calcium transients resulting from synaptic activation of calcium-permeable AMPARs or from slower NMDAR-mediated currents resulting from spillover of transmitter onto extrasynaptic sites. It will be interesting to discover whether the calcium signals resulting from these two routes of calcium entry are quantitatively different. It may be that a spatial separation of calcium-permeable AMPARs and NMDARs in some neuronal dendrites can provide for two distinct types of calcium signal in response to synaptic input.

\section{REFERENCES}

Armstrong DM, Rawson JA (1979) Activity patterns of cerebellar cortical neurones and climbing fibre afferents in the awake cat. J Physiol (Lond) 289:425-448.

Asztely F, Erdemli G, Kullman DM (1997) Extrasynaptic glutamate spillover in the hippocampus: dependence on temperature and the role of active glutamate uptake. Neuron 18:281-293.

Barbour B, Häusser M (1997) Intersynaptic diffusion of neurotransmitter. Trends Neurosci 20:377-384.

Barbour B, Keller BU, Llano I, Marty A (1994) Prolonged presence of glutamate during excitatory synaptic transmission to cerebellar Purkinje cells. Neuron 12:1331-1343.

Bekkers JM, Stevens CF (1989) NMDA and non-NMDA receptors are co-localized at individual excitatory synapses in cultured rat hippocampus. Nature 341:230-233.

Carter AG, Regehr WG (2000) Prolonged synaptic currents and glutamate spillover at the parallel fiber to stellate cell synapse. J Neurosci 20:4423-4434.

Chaudhry FA, Lehre KP, van Lookeren Campagne M, Ottersen OP, Danbolt NC, Storm-Mathisen J (1995) Glutamate transporters in glia membranes: highly differentiated localizations revealed by quantitative ultrastructural immunocytochemistry. Neuron 15:711-720.

Clark BA, Cull-Candy (1999) What is the role of NMDA receptors at cerebellar parallel fiber-stellate cell synapses? Soc Neurosci Abstr 25: 399.8 .

Clements JD, Westbrook GL (1991) Activation kinetics reveal the number of glutamate and glycine binding sites on the $N$-methyl-D-aspartate receptor. Neuron 7:605-613.

Clements JD, Lester RA, Tong G, Jahr CE, Westbrook GL (1992) The time course of glutamate in the synaptic cleft. Science 258:1498-1501.

Cull-Candy SG, Brickley SG, Misra C, Feldmeyer D, Momiyama A, Farrant M (1998) NMDA receptor diversity in the cerebellum: identification of subunits contributing to functional receptors. Neuropharmacology 37:1369-1380.

Diamond JS (2001) Neuronal glutamate transporters limit activation of NMDA receptors by neurotransmitter spillover on CA1 pyramidal cells. J Neurosci 21:8328-8338.

Eccles JC, Ito M, Szentágothai J (1967) The cerebellum as a neuronal machine. New York: Springer.

Farrant M, Cull-Candy SG (1991) Excitatory amino acid receptorchannels in Purkinje cells in thin cerebellar slices. Proc R Soc Lond B Biol Sci 244:179-184.

Furuta A, Martin LJ, Lin C-LG, Dykes-Hoberg M, Rothstein JD (1997) Cellular and synaptic localization of the neuronal glutamate transporters excitatory amino acid transporter 3 and 4 . Neuroscience 81:1031-1042.

Geiger JR, Lubke J, Roth A, Frotscher M, Jonas P (1997) Submillisecond AMPA receptor-mediated signaling at a principal neuroninterneuron synapse. Neuron 18:1009-1023.

Glitsch M, Marty A (1999) Presynaptic effects of NMDA in cerebellar Purkinje cells and interneurons. J Neurosci 19:511-519.

Griffiths R, Dunlop J, Gorman A, Senior J, Grieve A (1994) L-transPyrrolidine-2, 4-dicarboxylate and cis-1-aminocyclobutane-1, 3-dicarboxylate behave as transportable, competitive inhibitors of the high affinity glutamate transporters. Biochem Pharmacol 47:267-274.

Haas K, Cline H, Malinow R (1998) No change in NMDA receptormediated response rise-time during development: evidence against transmitter spillover. Neuropharmacology 37:1393-1398.

Hardingham NR, Larkman AU (1998) The reliability of excitatory synaptic transmission in slices of rat visual cortex in vitro is temperature dependent. J Physiol (Lond) 507:249-256.

Häusser M, Clark BA (1997) Tonic synaptic inhibition modulates neuronal output pattern and spatiotemporal integration. Neuron 19:665-678.

Hille B (1992) Ionic channels of excitable membranes. Sunderland, MA: Sinauer.

Holmes WR (1995) Modeling the effect of glutamate diffusion and uptake on NMDA and non-NMDA receptor saturation. Biophys $\mathrm{J}$ 69:1734-1747.

Isaac JT, Crair MC, Nicoll RA, Malenka RC (1997) Silent synapses during development of thalamocortical inputs. Neuron 18:269-280.

Jones KA, Baughman RW (1991) Both NMDA and non-NMDA subtypes of glutamate receptor are concentrated at synapses on cerebral cortical neurons in culture. Neuron 7:593-603.

Kharazia VN, Phend KD, Rustioni A, Weinberg RJ (1996) EM colocalization of AMPA and NMDA receptor subunits at synapses in rat cerebral cortex. Neurosci Lett 210:37-40.

Koch HP, Kavanaugh MP, Esslinger CS, Zerangue N, Humphrey JM, Amara SG, Chamberlain AR, Bridges RJ (1999) Differentiation of 
substrate and nonsubstrate inhibitors of the high affinity, sodium dependent glutamate transporters. Mol Pharmacol 56:1095-1104.

Kreitzer AC, Regehr WG (2000) Modulation of transmission during trains at a cerebellar synapse. J Neurosci 20:1348-1357.

Kutsuwada T, Kashiwabuchi N, Mori H, Sakimura K, Kushiya E, Araki K, Meguro H, Masaki H, Kumanishi T, Arakawa M, Mishina M (1992) Molecular diversity of the NMDA receptor channel. Nature 358:36-41.

Lester RA, Quarum ML, Parker JD, Weber E, Jahr CE (1989) Interaction of 6-cyano-7-nitroquinoxaline-2, 3-dione with the $N$-methyl-Daspartate receptor-associated glycine binding site. Mol Pharmacol 35:565-570.

Lester RA, Clements JD, Wesbrook GL, Jahr CE (1990) Channel kinetics determine the time course of NMDA receptor-mediated synaptic currents. Nature 346:565-567.

Li P, Zhuo M (1998) Silent glutamatergic synapses and nociception in mammalian spinal cord. Nature 393:695-698.

Liu S-Q, Cull-Candy SG (2000) Synaptic activity at calcium-permeable AMPA receptors induces a switch in receptor subtype. Nature 405:454-458.

Llano I, Marty A, Armstrong CM, Konnerth A (1991) Synaptic- and agonist-induced excitatory currents of Purkinje cells in rat cerebellar slices. J Physiol (Lond) 434:183-213.

Malinow R, Mainen ZF, Hayashi Y (2000) LTP mechanisms: from silence to four-lane traffic. Curr Opin Neurobiol 10:352-357.

Mann-Metzer P, Yarom Y (1999) Electrotonic coupling interacts with intrinsic properties to generate synchronized activity in cerebellar networks of inhibitory interneurons. J Neurosci 19:3298-3306.

Momiyama A, Feldmeyer D, Cull-Candy SG (1996) Identification of a native low-conductance NMDA channel with reduced sensitivity to $\mathrm{Mg}^{2+}$ in rat central neurones. J Physiol (Lond) 494:479-492.

Otis TS, Wu YC, Trussell LO (1996) Delayed clearance of transmitter and the role of glutamate transporters at synapses with multiple release sites. J Neurosci 16:1634-1644.

Palay S, Chan-Palay V (1974) Cerebellar cortex: cytology and organisation. New York: Springer

Perkel DJ, Hestrin S, Sah P, Nicoll RA (1990) Excitatory synaptic currents in Purkinje cells. Proc R Soc Lond B Biol Sci 241:116-121.

Racca C, Stephenson FA, Streit P, Roberts JD, Somogyi P (2000) NMDA receptor content of synapses in stratum radiatum of the hippocampal CA1 area. J Neurosci 20:2512-2522.

Rosenmund C, Feltz A, Westbrook GL (1995) Calcium-dependent inactivation of synaptic NMDA receptors in hippocampal neurons. J Neurophysiol 73:427-430.

Rumbaugh G, Vicini S (1999) Distinct synaptic and extrasynaptic NMDA receptors in developing cerebellar granule neurons. J Neurosci 19:10603-10610.
Rumpel S, Hatt H, Gottman K (1998) Silent synapses in the developing rat visual cortex: evidence for postsynaptic expression of synaptic plasticity. J Neurosci 18:8863-8874.

Rusakov DA, Kullman DM, Stewart MG (1999) Hippocampal synapses: do they talk to their neighbours? Trends Neurosci 22:382-388.

Shimamoto K, Lebrun B, Yasuda-Kamatani Y, Sakaitani M, Shigeri Y, Yumoto N, Nakajima T (1998) DL-threo-beta-benzyloxyaspartate, a potent blocker of excitatory amino acid transporters. Mol Pharmacol 53:195-201.

Silver RA, Traynelis SF, Cull-Candy SG (1992) Rapid-time-course miniature and evoked excitatory currents at cerebellar synapses in situ. Nature 355:163-166.

Stuart GJ, Dodt H-U, Sakmann B (1993) Patch-clamp recordings from the soma and dendrites of neurons in brain slices using infrared video microscopy. Pflügers Arch 423:511-518.

Takumi Y, Matsubara A, Rinvik E, Ottersen OP (1999) The arrangement of glutamate receptors at excitatory synapses. Ann NY Acad Sci $868: 474-482$.

Taschenberger H, Engert F, Grantyn R (1995) Synaptic current kinetics in a solely AMPA-receptor-operated glutamatergic synapse formed by rat retinal ganglion neurons. J Neurophysiol 74:1123-1136.

Tovar KR, Westbrook GL (1999) The incorporation of NMDA receptors with a distinct subunit composition at nascent hippocampal synapses in vitro. J Neurosci 19:4180-4188.

Umemiya M, Senda M, Murphy TH (1999) Behaviour of NMDA and AMPA receptor-mediated miniature EPSCs at rat cortical neuron synapses identified by calcium imaging. J Physiol (Lond) 521:113-122.

Van der Kloot W, Molgo J (1994) Quantal acetylcholine release at the vertebrate neuromuscular junction. Physiol Rev 74:899-991.

Volterra A, Bezzi P, Rizzini BL, Trotti D, Ullensvang K, Danbolt NC, Racagni G (1996) The competitive transport inhibitor L-transpyrrolidine-2, 4-dicarboxylate triggers excitotoxicity in rat cortical neuron-astrocyte co-cultures via glutamate release rather than uptake inhibition. Eur J Neurosci 8:2019-2028.

Waagepetersen HS, Shimamoto K, Schousboe A (2001) Comparison of effects of DL-threo-beta-benzyloxyaspartate (DL-TBOA) and L-transpyrrolidine-2,4-dicarboxylate $(t-2,4$-PDC) on uptake and release of $[\mathrm{H}]^{3}$ D-aspartate in astrocytes and glutamatergic neurons. Neurochem Res 26 661-666.

Wadiche JI, Kavanaugh MP (1998) Macroscopic and microscopic properties of a cloned glutamate transporter/chloride channel. J Neurosci 18:7650-7661.

Wu G, Malinow R, Cline H (1996) Maturation of a central glutamatergic synapse. Science 274:972-976. 